Solving Dirichlet problems

numerically using the FeynmanKac representation

\title{
Report
}

Author(s):

Buchmann, Fabian M.; Petersen, Wesley P.

Publication date:

2002-02

Permanent link:

https://doi.org/10.3929/ethz-a-004339274

Rights / license:

In Copyright - Non-Commercial Use Permitted

Originally published in:

SAM Research Report 2002-01 


\section{Solving Dirichlet problems numerically using the Feynman-Kac representation}

F.M. Buchmann and W.P. Petersen

Research Report No. 2002-01

February 2002

Seminar für Angewandte Mathematik

Eidgenössische Technische Hochschule CH-8092 Zürich

Switzerland 


\title{
Solving Dirichlet problems numerically using the Feynman-Kac representation
}

\section{F.M. Buchmann and W.P. Petersen}

Seminar für Angewandte Mathematik

Eidgenössische Technische Hochschule

CH-8092 Zürich

Switzerland

Research Report No. 2002-01

February 2002

\begin{abstract}
In this paper we study numerical solutions of the Dirichlet problem in high dimensions using the Feynman-Kac representation. What is involved are Monte-Carlo simulations of stochastic differential equations and algorithms to accurately determine exit times and process values at the boundary. It is assumed that the radius of curvature of the boundary is much larger than the square root of the step-size. We find that the canonical $\mathcal{O}\left(N^{-1 / 2}\right)$ behavior of statistical errors as a function of the sample size $N$ holds regardless of the dimension $n$ of the space. In fact, the coefficient of $N^{-1 / 2}$ seems to actually decrease with $n$. Additionally, acceptance ratios for finding the boundary become less sensitive to the time step size in higher dimensions. The walk on cubes method, wherein the model increments of Brownian motion are three-point random variables, is of particular interest. Comparisons are made between this walk-on-cubes method, Milstein's walk on spheres, and a simpler 2-point method. Our examples have hyperspherical domains up to $n=64$ dimensions.
\end{abstract}

Keywords: Stochastic differential equations, Monte-Carlo, Dirichlet, PDE

Subject Classification: 60J65, 65C30, 65N99 


\section{Introduction.}

In many applications, the dimension of the underlying space can be much larger than three. Solutions of partial differential equations in three space can be an industrial project, while in higher dimensions this is even more formidable. In financial applications or many body simulations, the underlying space may be 50 or more. Monte-Carlo simulation thus becomes most attractive when the dimension $n$ of the configuration space is high. In this paper, we explore the Dirichlet problem in high dimensions using variants of the Feynman-Kac representation (e.g. [5]). What is involved is to simulate some stochastic differential equations, functionals of which form the Feynman-Kac formula. In simulation, most of the functionals turn out to be simple averages of multiple realizations of functions of independent sample paths. For the Dirichlet problem, the boundary values make the solution unique. Finding the boundary and determining the exit time of the diffusion process when it crosses the boundary are the main tasks.

In a basic form, the Feynman-Kac representation for the Dirichlet problem is follows. Let $L$ be a linear second order differential operator

$$
L u=\frac{1}{2} \triangle u+\sum_{i=1}^{n} b_{i}(x) \partial_{i} u+c(x) u=-g(x) .
$$

In this study, we consider only the case that the potential $c(x) \leq 0$ and that $b, c, g$ are smooth and satisfy some Lipshitz growth conditions. The Dirichlet problem is to find $u(x)$ satisfying the above PDE (1.1), while at the boundary of a bounded domain $D, u(x)=\psi(x)$, when $x \in \partial D$. The probabilistic representation for the solution $u$ is the Feynman-Kac formula,

$$
u(x)=\mathbb{E} \psi(X(\tau)) e^{\int_{0}^{\tau} c(X(s)) d s}+\mathbb{E} \int_{0}^{\tau} g(X(t)) e^{\int_{0}^{t} c(X(s)) d s} d t,
$$

where $\tau$ is the exit time from domain $D$ of a process $X(t)$ whose initial value is $X(0)=x$ and which satisfies a stochastic differential equation (SDE)

$$
d X(t)=b(X) d t+d W .
$$

In this equation, $W$ is an $n$-dimensional Brownian motion, and $b$ is the vector which appears in the PDE (1.1). The exit time is $\tau=\inf \{t: X(t) \in \partial D\}$, i.e. the first time the process $X$ crosses the boundary. Two difficulties present themselves: (i) finding the process value $X(\tau)$ accurately at the boundary, and (ii) making sure that this value does not follow an excursion, that is, $X(t)$ cannot leave the domain and come back. In practice, one writes $Y(t)=\exp \int_{0}^{t} c(X(s)) d s$ and computes $Y$ from the differential equation $d Y=c(X) Y d t$.

If the numerical representation of the increments of $W$ is Gaussian, controlling both problems ((i) and (ii)) is very hard because these increments are unbounded. Three bounded approximations to these increments are known and used:

\section{- A simple two point:}

$$
\Delta W^{i}= \pm \sqrt{h} \text { each with probability } 1 / 2
$$


- A walk on spheres, where $\Delta W$ is uniformly (isotropically) distributed on an $n$-sphere. In this case, the calculus for the SDE is modified for the conditions that the process value is always on a hyperspherical surface.

- A walk on cubes (3-point, (3.5)), wherein

$$
\begin{aligned}
& \Delta W^{i}= \pm \sqrt{3 h} \text { each with probability } 1 / 6 \\
& \Delta W^{i}=0 \text { with probability } 2 / 3
\end{aligned}
$$

The moment conditions

$$
\begin{aligned}
\mathbb{E} \Delta W^{i} & =0, \\
\mathbb{E} \Delta W^{i} \Delta W^{j} & =h \delta_{i j}, \\
\mathbb{E} \Delta W^{i} \Delta W^{j} \Delta W^{k} & =0, \\
\mathbb{E} \Delta W^{i} \Delta W^{j} \Delta W^{k} \Delta W^{l} & =h^{2}\left\{\delta_{i j} \delta_{k l}+\delta_{i k} \delta_{j l}+\delta_{i l} \delta_{j k}\right\}
\end{aligned}
$$

are satisfied to $\mathcal{O}\left(h^{2}\right)$, but the next even order relations, $\mathcal{O}\left(h^{3}\right)$, are not correct. This walk on cubes gives a better representation than two point or uniform distributions on spheres for the 1-D distribution function $p\left(\Delta X_{i}\right)=$ $\sqrt{1 / 2 \pi h} \cdot \exp \left(-\Delta X_{i}^{2} / 2 h\right)$

$$
p\left(\Delta X_{i}\right) \approx \frac{1}{6} \delta\left(\Delta X_{i}+\sqrt{3 h}\right)+\frac{1}{6} \delta\left(\Delta X_{i}-\sqrt{3 h}\right)+\frac{2}{3} \delta\left(\Delta X_{i}\right) .
$$

In the 2-point and walk on cubes cases, the joint distribution for all the $n$ $\Delta X$ 's isn't isotropic. For an arbitrarily oriented boundary, this lack of isotropy introduces errors. It is possible to remove this lack of isotropy by choosing a vector increment $\Delta X=\left\{\Delta X_{1}, \Delta X_{2}, \ldots, \Delta X_{n}\right\}$ and rotating it by a random orthogonal matrix, say $Q$. That is, for any unit vector $e$, the set $Q e$ will be uniformly distributed on surface of a unit $n$-sphere. In 2 or 3 dimensions, generating such $Q$ is straightforward, but in higher dimensions this might be more tedious. In this study, we do not attempt to correct for this lack of isotropy, but note that the problem exists.

In this paper, we study test problems in high dimensions in a hyperspherical domain. This domain will be called $D$. Additionally, two border areas of $D$ are: $\Gamma_{T}$ is a boundary finding region, which we'll call the tangent layer; and a stopping region $\Gamma_{S}$ where the discrete Markov chain of our approximation terminates. These are subsets of $D, \Gamma_{S} \subset \Gamma_{T} \subset D$. Our basic test problem is

$$
\frac{1}{2} \triangle u(x)+g(x)=0
$$

where $g$ and the boundary values $\psi(x), x \in \partial D$ are chosen such that we know the solution (see section 4 ). This will enable us to examine the boundary finding strategy and study the models for bounded approximations to Brownian increments. Our particular goal is to see how the solutions behave as the dimension $n$ of the problem increases. 
Our notation is conventional, but the reader should note a perhaps inconsistent meaning of the stepsize $h$. When finding the boundary, the global stepsize $h$ is shrunk according to the distance to that boundary. When discussing the expected number of steps, and the order of accuracy, $h$ means the initial stepsize: $h$ when $X \in D \backslash \Gamma_{T}$. Within $\Gamma_{T}, h$ shrinks - we do not allow excursions. In the simulations, the expectation $\mathbb{E} \phi$ for some $\phi$ is computed by the mean $\mathbb{E} \phi \rightarrow N^{-1} \sum_{i}^{N} \phi^{(i)}$ over some sample of $N \phi$ 's.

\section{Exit time from the domain.}

We first examine a simple exit-time problem, in part to illustrate that even in a simple case, the analysis is non-trivial. The solution to the $n$-dimensional constant potential problem on an $n$-sphere with radius $r$

$$
\frac{1}{2} \triangle u-\lambda u=0
$$

where $u(x)=1$ when $|x|=r$ can be written using the Feynman-Kac formula. In this case it reads (equation 1.2)

$$
u(x)=\mathbb{E}_{x} e^{-\lambda \tau},
$$

where $\tau$ (the exit time) is the first $t$ for an $n$-dimensional Brownian motion $x+W(t)$ which begins at $x$ and $|x+W(\tau)|=r$. The solution $u(x)$ is the Laplace transform of the distribution function for the exit time $\tau$. In hyperspherical coordinates (see [4]) $\left(^{\prime}=d / d|x|\right)(2.1)$ is

$$
\frac{1}{2} u^{\prime \prime}+\frac{1}{2} \frac{n-1}{|x|} u^{\prime}-\lambda u=0
$$

The solution is relatively easily found by the transformation $v(|x|)=|x|^{\nu} u(|x|)$, where $\nu=n / 2-1$, from which one gets the equation for a modified Bessel function. The solution to $(2.1)$ is [7]

$$
u(x)=\frac{r^{\nu} I_{\nu}(\sqrt{2 \lambda} \cdot|x|)}{|x|^{\nu} I_{\nu}(\sqrt{2 \lambda} \cdot r)} .
$$

To invert the Laplace transform, we used the infinite product form of the modified Bessel function ([1]),

$$
I_{\nu}(t)=\frac{1}{\Gamma(\nu+1)}\left(\frac{t}{2}\right)^{\nu} \prod_{s=1}^{\infty}\left(1+\left(\frac{t}{j_{\nu, s}}\right)^{2}\right) .
$$

All the terms in the product for $u(x)$ are even and hence only contain $\lambda$, not its square root. We may apply the inversion formula by closing the contour in the left-half plane, with the poles $\lambda=-\frac{\left(j_{\nu, s}\right)^{2}}{2 r^{2}}$ appearing only on the negative real 
axis. Here, $j_{\nu, s}$, is the $s$-th real positive zero of the Bessel function $J_{\nu}$, where again $\nu=n / 2-1$. Our result for the distribution function is

$$
p(x, \tau)=\frac{1}{r^{2}}\left(\frac{r}{|x|}\right)^{\nu} \sum_{s=1}^{\infty} \frac{j_{\nu, s} J_{\nu}\left(j_{\nu, s} \frac{|x|}{r}\right)}{J_{\nu+1}\left(j_{\nu, s}\right)} e^{-\frac{\left(j_{\nu, s}\right)^{2}}{2 r^{2}} \tau}
$$

which for $x=0$ (the origin) reduces to

$$
p(0, \tau)=\frac{2}{\Gamma\left(\frac{n}{2}\right) r^{2}} \sum_{s=1}^{\infty} \frac{\left(\frac{j_{\nu, s}}{2}\right)^{\frac{n}{2}}}{J_{\nu+1}\left(j_{\nu, s}\right)} e^{-\frac{\left(j_{\nu, s}\right)^{2}}{2 r^{2}} \tau}
$$

Both series converges for all $\tau>0$ because the denominators never vanish for finite $s$ by Lomelin's interleaving theorem (see Watson [15]). The series for moments $\mathbb{E}_{0} \tau^{m}$ of (2.3) converges only if $n / 2-m-1 \leq 0$ because the amplitude of the Bessel functions in the denominators decrease for large $s$ as $\mathcal{O}\left(s^{-1 / 2}\right)$ and the signs alternate. Hence, there are "n.a." (not available) entries in Table 2.2. To check our result (2.2), we computed the moments $\mathbb{E}_{0} \tau^{m}$ for $m=1 \ldots 4$ and compared these moments to Dynkin's [3] recursive procedure. Following Milstein [10], who computed the moments $\mathbb{E}_{x} \tau^{m}$ for $m=1,2,3$, we use Dynkin's idea [3] where

$$
h_{m}(x)=\mathbb{E}_{x} \tau^{m} .
$$

Then $h_{m}$ for $m \geq 1$ satisfies the equation

$$
\frac{1}{2} \triangle h_{m}+m h_{m-1}=0
$$

with $h_{m}(x)=0$ when $|x|=r$, although $h_{0}=1$. $h_{m}$ is an $m$-th order homogeneous polynomial in $\left(|x|^{2}, r^{2}\right)$. The first 4 solutions for $h_{m}(x)$ are given in Table 2.1. Table 2.2 shows our comparisons. One obvious feature of these results is that as $n$ increases, the distributions sharpen around the expected value $\mathbb{E} \tau$. That is,

$$
\frac{\operatorname{var}(\tau)}{\left(\mathbb{E}_{0} \tau\right)^{2}}=\frac{2}{n+2}
$$

\subsection{The number of steps to find boundary.}

Our basic scheme is to set up a discrete stochastic process, $\left\{X_{k}: X_{0}=x, k=\right.$ $\left.0 \ldots \nu_{x}\right\}$, which weakly approximates a Brownian motion. If the initial point $x \in D \backslash \Gamma_{T}$, our increments $\Delta X$ will depend on the initial stepsize $h$. If $X$ (including the case $x \in \Gamma_{T}$ ) is in the tangent layer $\Gamma_{T}$, the stepsize is shrunk so that the largest bounded increment $X \rightarrow X+\Delta X$ could at most take $X$ to the boundary $\partial D$. We now show that for bounded approximations to Gaussian increments, the number of steps is $\mathcal{O}\left(h^{-1}\right)$. After [10], we define

$$
A \nu(X)=\mathbb{E} \nu(X+\Delta X)-\nu(X) .
$$


Table 2.1: Solutions to Dynkin's equations for moments

\begin{tabular}{||c|c||}
\hline$h_{m}$ & polynomial \\
\hline$h_{1}$ & $\frac{-|x|^{2}+r^{2}}{n}$ \\
\hline$h_{2}$ & $\frac{|x|^{4}}{n(n+2)}-\frac{2|x|^{2} r^{2}}{n^{2}}+\frac{(n+4) r^{4}}{n^{2}(n+2)}$ \\
\hline$h_{3}$ & $-\frac{|x|^{6}}{n(n+2)(n+4)}+\frac{3|x|^{4} r^{2}}{n^{2}(n+2)}-\frac{3(n+4)|x|^{2} r^{4}}{n^{3}(n+2)}+\frac{\left(n^{2}+12 n+48\right) r^{6}}{n^{3}(n+2)(n+4)}$ \\
\hline$h_{4}$ & $\frac{|x|^{8}}{n(n+2)(n+4)(n+6)}-\frac{4|x|^{6} r^{2}}{n^{2}(n+2)(n+4)}+\frac{6(n+4)|x|^{4} r^{4}}{n^{3}(n+2)^{2}}$ \\
& $-\frac{4\left(n^{2}+12 n+48\right)|x|^{2} r^{6}}{n^{4}(n+2)(n+4)}+\frac{\left(n^{4}+26 n^{3}+288 n^{2}+1536 n+2304\right) r^{8}}{n^{4}(n+2)^{2}(n+4)(n+6)}$ \\
\hline
\end{tabular}

Table 2.2: Comparison of integrating series (2.2) (100 terms) for $\mathbb{E}_{0} \tau^{m}=\int p \tau^{m} d \tau$ vs. the recursive procedure of Dynkin: $\mathbb{E}_{0} \tau^{m}=h_{m}(0)$. Entries marked n.a. are "not available" because of lack of numerical convergence: see the explanation following (2.2).

\begin{tabular}{||c|l|l|l|l||}
\hline Moment & $\mathrm{n}=2$ & $\mathrm{n}=3$ & $\mathrm{n}=4$ & $\mathrm{n}=5$ \\
\hline $\mathbb{E} 1$ & 1.0000 & 1.0000 & 1.0000 & 1.000 \\
$\mathbb{E} 1$ Series & 0.9900 & n.a. & n.a. & n.a. \\
\hline $\mathbb{E} \tau$ Dynkin & 0.5000 & 0.3333 & 0.2500 & 0.2000 \\
$\mathbb{E} \tau$ Series & 0.4999 & 0.3333 & 0.2498 & 0.1979 \\
\hline $\mathbb{E} \tau^{2}$ Dynkin & 0.3750 & 0.1556 & 0.0833 & 0.0514 \\
$\mathbb{E} \tau^{2}$ Series & 0.3750 & 0.1556 & 0.0833 & 0.0514 \\
\hline $\mathbb{E} \tau^{3}$ Dynkin & 0.3958 & 0.0984 & 0.0366 & 0.0169 \\
$\mathbb{E} \tau^{3}$ Series & 0.3958 & 0.0984 & 0.0366 & 0.0169 \\
\hline $\mathbb{E} \tau^{4}$ Dynkin & 0.5495 & 0.0806 & 0.0203 & 0.0069 \\
$\mathbb{E} \tau^{4}$ Series & 0.5495 & 0.0806 & 0.0203 & 0.0069 \\
\hline
\end{tabular}


And from Wentzell [16], we are looking for a potential function $\nu(X)$ which satisfies the criteria

$$
\begin{array}{lrr}
\nu(X) \geq 0 & \text { when } & X \in D \backslash \Gamma_{S} \\
\nu(X)=0 & \text { for } & X \in \Gamma_{S},
\end{array}
$$

and such that when $f(X) \geq 1$ in the equation

$$
A \nu=\mathbb{E} \nu(X+\Delta X)-\nu(X)=-f(X) \quad \text { when } \quad X \in D \backslash \Gamma_{S},
$$

then

$$
\text { \# steps }=\nu_{x} \leq \nu(x) .
$$

For any Markov chain, $\left\{X_{k}\right\}$, beginning at $X_{0}=x$,

$$
\begin{aligned}
\mathbb{E}_{1} \nu\left(X_{1}\right)-\nu(x) & =-f(x) \\
\mathbb{E}_{2} \nu\left(X_{2}\right)-\nu\left(X_{1}\right) & =-f\left(X_{1}\right) \\
\mathbb{E}_{3} \nu\left(X_{3}\right)-\nu\left(X_{2}\right) & =-f\left(X_{2}\right) \\
\cdots & \\
\underbrace{\mathbb{E}_{\nu_{x}} \nu\left(X_{\nu_{x}}\right)}_{0, X_{\nu_{x}} \in \Gamma_{S}}-\nu\left(X_{\nu_{x}-1}\right) & =-f\left(X_{\nu_{x}-1}\right) .
\end{aligned}
$$

Here, $\mathbb{E}_{k}$ means expectation with respect to the independent increment $\Delta X_{k}=$ $X_{k}-X_{k-1}: \mathbb{E}_{k} \nu\left(X_{k}\right)=\mathbb{E}\left[\nu\left(X_{k}\right) \mid X_{k-1}\right]$. Working backwards, the unique solution to the boundary value problem

$$
\begin{array}{lcr}
A \nu(X)=-f(X) & \text { when } & X \in D \backslash \Gamma_{S}, \\
\nu(X)=0 & \text { for } & X \in \Gamma_{S},
\end{array}
$$

is $([10],[16])$

$$
\nu(x)=\mathbb{E} \sum_{k=0}^{\nu_{x}-1} f\left(X_{k}\right) .
$$

So if $f \geq 1$, then $\nu_{x} \leq \nu(x)$.

\subsection{One dimensional case.}

Although it is a bit special, the one dimensional situation is edifying. Consider the domain $-L \leq x \leq L$ with a tangent layer

$$
\Gamma_{T}=\{-L \leq X<-L+\sqrt{3 h}\} \cup\{L-\sqrt{3 h} \leq X<L\}
$$

and stopping layer $\Gamma_{S}$,

$$
\Gamma_{S}=\left\{-L \leq X<-L+\delta_{S}\right\} \cup\left\{L-\delta_{S} \leq X<L\right\} .
$$

For a two point approximation $(\Delta X= \pm \sqrt{h}), \sqrt{3 h} \rightarrow \sqrt{h}$ in the first definition. The width of the stopping layer is given by $\delta_{S}=h^{2}$, or $\delta_{S}=h$ for a two point approximation. We want to find a potential $\nu(x)$ which bounds the number of steps of the chain $\left\{X_{0}=x, X_{1}, \ldots, X_{\nu_{x}} \in \Gamma_{S}\right\}$, with the properties 
- $\nu(x)$ increases as $x$ moves away from the boundaries $-L, L$, and

- $\nu\left(|X+L| \leq \delta_{S}\right)=0$, or $\nu\left(|X-L| \leq \delta_{S}\right)=0$ for left or right boundary, respectively.

Let $\epsilon$ be a bounded step. In the 2-point, or simple random walk $(\Delta X=\epsilon=$ $\pm \sqrt{h})$

$$
A \nu(X)=\frac{1}{2}(\nu(X+\epsilon)+\nu(X-\epsilon))-\nu(X) .
$$

For the 3-point (walk-on-cubes) case $(\Delta X=\epsilon= \pm \sqrt{3 h}$, or 0$)$,

$$
\begin{aligned}
A \nu(X) & =\frac{1}{6}(\nu(X+\epsilon)+\nu(X-\epsilon))+\frac{2}{3} \nu(X)-\nu(X) \\
& =\frac{1}{3}\left(\frac{1}{2} \nu(X+\epsilon)+\frac{1}{2} \nu(X-\epsilon)-\nu(X)\right)
\end{aligned}
$$

It is important to notice that the scaling of $\nu$ must be increased if $A \nu \leq-1$ in the 3 -point (walk-on-cubes) case. Indeed, for a better representation of the radial distribution, the acceptance decreases by $1 / 3$ in this case. A walk on spheres is the same as the 2-point case in one dimension. For this, the conditions that $A \nu<0$ are thus the same for all three models for the increment $\Delta X=\epsilon: \nu \geq 0$ must decrease to zero toward the stopping layer and turn down in the interior. There are two cases to examine

$$
\begin{array}{lll}
\text { (a) } X \in D \backslash \Gamma_{S} \quad \text { and both } & X \pm \epsilon \notin \Gamma_{S} \\
\text { (b) } X \in D \backslash \Gamma_{S} & \text { but one of } & X+\epsilon \in \Gamma_{S} \text {, or } X-\epsilon \in \Gamma_{S} .
\end{array}
$$

In the interior case (a), for $A \nu(X)=\mathbb{E} \nu(X+\Delta X)-\nu(X)$ to be negative, we see from the remainder form of Taylor's series (with numbers $0 \leq \eta, \gamma \leq 1$ ),

$$
\begin{aligned}
\mathbb{E} \nu(X+\Delta X)-\nu(X) & =\frac{1}{2}\{\nu(X+\epsilon)+\nu(X-\epsilon)\}-\nu(X) \\
& =\frac{1}{4}\left\{\nu^{\prime \prime}(X+\eta \epsilon) \epsilon^{2}+\nu^{\prime \prime}(X-\gamma \epsilon)\right\} \epsilon^{2},
\end{aligned}
$$

that it suffices for $\nu^{\prime \prime}(Y)<0$ for $Y \in D \backslash \Gamma_{S}$. Case (b) is that one of the possible steps ends up in $\Gamma_{S}$. We look only at the right boundary $(\nu(X+\epsilon)=0)$ since the left one is similar, (again, $0 \leq \eta \leq 1$ )

$$
A \nu(X)=\frac{1}{2} \nu(X-\epsilon)-\nu(X)=-\frac{1}{2} \nu^{\prime}(X) \epsilon+\frac{1}{4} \nu^{\prime \prime}(X+\eta \epsilon) \epsilon^{2}-\frac{1}{2} \nu(X) .
$$

For the bounded step, sufficient conditions for $\nu$ to be a bounding potential when $X \in D \backslash \Gamma_{S}$ are

- $\nu(X)$ vanishes when $X$ hits $\Gamma_{S}$

- $\nu(X)$ increases as $X$ moves away from $\Gamma_{S}$. That is, when $|X+\Delta X-\partial D|>$ $|X-\partial D|$ we have $\Delta X \cdot \nabla \nu(X)<0$. This is a condition on $\Delta X$ : it takes $X \rightarrow X+\Delta X$ into the interior, not towards the boundary. 
- $\nu(X)$ turns down: $\triangle \nu(X)<0$ for all $X \in D \backslash \Gamma_{S}$.

The above conditions remain valid for the $n$-dimensional case, hence the notation. In $n$-D, the condition $(\Delta \nu<0)$ follows from the Itô formula:

$$
\begin{aligned}
\nu(X+\Delta X) & =\nu(X)+\int_{0}^{h} \nabla \nu(X(t)) \cdot d X(t)+\frac{1}{2} \int_{0}^{h} \triangle \nu(X(t)) d t, \text { so } \\
A \nu & =\frac{1}{2} \int_{0}^{h} \mathbb{E} \triangle \nu(X(t)) d t .
\end{aligned}
$$

Hence, if $\triangle \nu(X(t))<0$ all along the path from $X \rightarrow X+\Delta X, A \nu<0$. In our one dimensional symmetric example, such a potential [10] begins with

$$
\begin{aligned}
& \nu_{2}(X)=c_{0}\left(\log \frac{\sqrt{3 h}}{\delta_{S}}+1\right) \text { when }|X|<|L|-\sqrt{3 h}, \\
& \nu_{2}(X)=c_{0}\left(\log \frac{\left|L-\delta_{S}-\right| X||}{\delta_{S}}+1\right) \quad \text { when }|L|-\sqrt{3 h} \leq|X|<|L|-\delta_{S}, \\
& \nu_{2}(X)=0 \text { when }|X| \geq|L|-\delta_{S} .
\end{aligned}
$$

Then

$$
\begin{aligned}
& \nu(x)=\frac{1}{2 h}\left(L^{2}-\delta_{S}^{2}-x^{2}\right)+\nu_{2}(X) \text { when } X \in D \backslash \Gamma_{S}, \\
& \nu(x)=0 \text { when } \quad X \in \Gamma_{S},
\end{aligned}
$$

where $c_{0}=c_{0}(h)$ is a non-zero constant which may be chosen large enough that $A \nu_{2} \leq-1$ (see [10]).

\subsection{Potential $\nu$ for bounded approximations to Gaussian increments.}

For a bounded approximation to Gaussian increments, we define the following function:

$$
\begin{array}{lll}
\nu_{0}(X)=L^{2}-|X|^{2} & \text { when } & X \in D \backslash \Gamma_{S}, \\
\nu_{0}(X)=0 & \text { for } \quad & X \in \Gamma_{S} .
\end{array}
$$

We may choose $L$ large enough that $\nu_{0} \geq 0$ for a bounded domain $D$. Additionally,

$$
\begin{aligned}
\nu_{h}(X) & =-\mathcal{K}_{0} \\
& =-\int_{\left(\partial \Gamma_{S}\right) \backslash(\partial D)} k(x, y) \nu_{0}(y) d S_{y},
\end{aligned}
$$

with $d S_{y}$ is the outward oriented area increment of the inner surface of the exit layer $\Gamma_{S}$, that is $X \in\left(\partial \Gamma_{S}\right) \backslash(\partial D)$, and $k(x, y)$ is the Green function for the Laplace operator. For a sphere, $k(x, y)$ is the Poisson kernel: see Doob [2] chapter II, section 1. The function $\nu_{h}(X)$ is harmonic in $D \backslash \Gamma_{S}$ and is equal to 
$-\nu_{0}(X)$ when $X \in\left(\partial \Gamma_{S}\right) \backslash(\partial D)$. We now begin construction of $\nu$ which will consist of three terms: the first two $\nu_{0}, \nu_{h}$ are interior terms, and the last will be for the tangent layer.

Lemma 2.1. For $X \in D \backslash \Gamma_{S}$,

$$
\nu_{0}+\nu_{h} \geq 0
$$

Proof. With $L$ chosen to be large enough, $\nu_{0} \geq 0$, and $\nu_{0}$ is subharmonic. By construction, $\nu_{h}(X)$ is harmonic and

$$
-\nu_{h}(X)=\nu_{0}(X) \text { when } X \in\left(\partial \Gamma_{S}\right) \backslash(\partial D) .
$$

Thus, from Doob $([2])$, chapter II, section $7, \nu_{0}+\nu_{h} \geq 0$ as desired.

We note that $\nu_{0}+\nu_{h}$ reaches its maximum in the interior because $\nu_{0}+\nu_{h} \geq 0$ when $X \in D \backslash \Gamma_{S}$, is not identically zero, but $\nu_{0}+\nu_{h}=0$ on the exit layer surface $X \in\left(\partial \Gamma_{S}\right) \backslash(\partial D)$.

LEMma 2.2. The function

$$
\nu_{1}(X)=\frac{1}{2 n h}\left(\nu_{0}+\nu_{h}\right)
$$

satisfies

$$
\mathbb{E} \nu_{1}(X+\Delta X)-\nu_{1}(X)=-1
$$

Proof. In our case, $X$ is a Brownian motion: $X=x+W(t)$. We use the rule, for even $m$,

$\mathbb{E}\left\{\Delta X_{i_{1}} \Delta X_{i_{2}} \ldots \Delta X_{i_{m}}\right\}=h^{m / 2}$. pairwise permutations of $\left\{\delta_{i_{1}, i_{2}} \ldots \delta_{i_{m-1}, i_{m}}\right\}$, whereas odd numbers of $\Delta X$ vanish, to get

$$
\mathbb{E} \nu_{1}(X+\Delta X)-\nu_{1}(X)=\frac{h}{2} \Delta \nu_{1}(X)+\frac{3 h^{2}}{4 !} \Delta^{2} \nu_{1}(X)+\ldots
$$

Formally,

$$
\mathbb{E} \nu_{1}(X+\Delta X)-\nu_{1}(X)=e^{\frac{h}{2} \triangle} \nu_{1}(X)-\nu_{1}(X) \text {. }
$$

Now,

$$
\frac{1}{2 n h} \triangle \nu_{0}(X)=-1,
$$

and because $\nu_{h}$ is harmonic

$$
\triangle \nu_{h}(X)=0,
$$

we get all the higher order terms

$$
\begin{aligned}
& \triangle^{k+1} \nu_{0}=\triangle^{k}(-1)=0, \text { and } \\
& \triangle^{k+1} \nu_{1}=\triangle^{k}(0)=0,
\end{aligned}
$$


for the desired result. Namely, all the $\mathcal{O}\left(h^{2}\right)$ and higher terms of (2.5) vanish leaving only (2.6).

We now need a contribution to $\nu(x)$ [10] appropriate for the boundary finding tangent layer $\Gamma_{T}$. When $X \in \Gamma_{T} \backslash \Gamma_{S}$, the time step is computed from the distance to the boundary. Where $\rho=\inf |X-\partial D|$ and $\delta_{S}=h^{2}$, let

$$
\begin{aligned}
& \nu_{2}=c_{0}\left(\log \frac{\sqrt{3 h}}{\delta_{S}}+1\right) \quad \text { when } \quad X \in D \backslash \Gamma_{T} \\
& \nu_{2}=c_{0}\left(\log \frac{\rho}{\delta_{S}}+1\right) \quad \text { when } \quad X \in \Gamma_{T} \backslash \Gamma_{S} \\
& \nu_{2}=0 \quad \text { for } \quad X \in \Gamma_{S} \text {. }
\end{aligned}
$$

The constant $c_{0}$ depends only on $n$ and $h$ and is large enough that $A \nu_{2} \leq-1$ [10]. This potential has the following properties [10]:
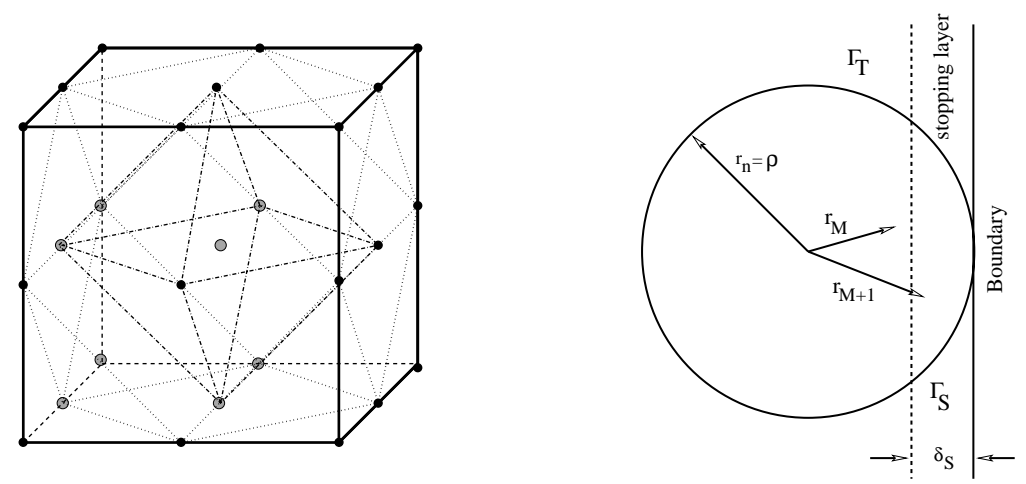

Figure 2.1: Nested $n$-polyhedra (left), stopping geometry (right)

Lemma 2.3. For $\rho \geq \delta_{S}$,

$\nu_{2}(\rho) \geq 0$,

$\Delta X \cdot \nabla \nu_{2} \leq 0$

i.e. $\nu_{2}$ is increasing if $\Delta X$ moves $X$ away from $\Gamma_{S}$,

$A \nu_{2}<$ constant $<0$.

Proof. That $\nu_{2}$ is positive is obvious. When $\Delta X$ moves $X$ away from the boundary $\partial D, \Delta X \cdot \nabla \nu_{2} \leq 0$ because $\log \rho / \delta_{S}$ increases. To show $A \nu_{2}<0$ is more difficult. Milstein [10] showed that for a ball of fixed size $\rho=|\Delta X|$, $A \nu_{2}<$ constant $<0$. We simplify the argument for the walk on cubes, namely we inscribe the polyhedra in spheres. Figure 2.1 illustrates the situation for $n=3$. In the general $n$ case, the possible points form a set of nested $n$-polyhedra 
whose radii are $r_{k}=\sqrt{3 k h}$ for $k=0 \ldots n$. The number of points at the vertices of these polyhedra at radius $r_{k}$ is given by by

$$
\# \text { points }_{k}=\left(\begin{array}{c}
n \\
k
\end{array}\right) 2^{k} .
$$

We weight these points on the surface of an $n$-ball of radius $r_{k}$ by the probability that these vertices have radius $r_{k}$, that is

$$
P_{k} \equiv P\left\{r_{k}=\sqrt{3 k h}\right\}=\frac{1}{3^{n}}\left(\begin{array}{c}
n \\
k
\end{array}\right) 2^{k},
$$

where $h=\frac{|\rho|^{2}}{3 n}$. Also,

$$
\sum_{k=0}^{n} P_{k}=(1 / 6+1 / 6+2 / 3)^{n}=1
$$

Now let $M \geq 0$ be the number of these radii $r_{k}<\rho-\delta_{S}, k=0 \ldots M$. The $k=0$ contribution is a point, so contributes nothing to $A \nu_{2}$. Thus, averaging over the weighted points as though they are equally distributed on the surfaces of the spheres at $r_{k}$ (for an arbitrarily oriented boundary at distance $\rho$ ),

$$
A \nu_{2}(X)=\sum_{k=1}^{M}+\sum_{k>M}^{n} \frac{P_{k}}{A_{k}} \int d S_{k} \nu_{2}\left(X+\Delta X_{k}\right)-P_{k} \nu_{2}(X) .
$$

Here, $A_{k}=\frac{2 \pi^{n / 2}}{\Gamma\left(\frac{n}{2}\right)} r_{k}^{n-1}$ is the surface area of the $n$-ball of radius $r_{k}$. For $k=1 \ldots M$, there is no overlap of the ball with the stopping layer (see Figure 2.1 ), so since $\triangle \nu_{2}<0$, the $k=1 \ldots M$ contributions to (2.7) are negative. The remaining $k>M$ are also negative and bounded away from zero by Milstein's theorem ([10]: his Lemmas 4.1, 4.2, and Theorem 4.1). Indeed, by our choice of stepsize, at least one, $r_{n}=\rho$, overlaps the stopping layer $\Gamma_{S}$. Thus $A \nu_{2}<$ const. $<0$. That is, the scaling $c_{0}$ is bounded.

It is important that when $M>0$, the acceptance ratio for stopping decreases. Finally, these three pieces give us the following.

Theorem 2.4. The potential $\nu(X)=\frac{1}{2 n h}\left(\nu_{0}(X)+\nu_{h}(X)\right)+\nu_{2}(X)=\nu_{1}(X)+$ $\nu_{2}(X)$ has the desired properties:

$$
\begin{array}{lcr}
\nu(X) \geq 0 & \text { when } & X \in D \backslash \Gamma_{S} \\
\nu(X)=0 & \text { if } & X \in \Gamma_{S} \\
\mathbb{E} \nu(X+\Delta X)-\nu(X) \leq-1 & \text { for } & X \in D \backslash \Gamma_{S}
\end{array}
$$

Proof. This follows directly from Lemmas 2.1, 2.2, and 2.3.

THEOREM 2.5. Where $h$ is the initial stepsize, the expected number of steps using these bounded approximations for Gaussian increments is $\mathcal{O}\left(h^{-1}\right)$. 
Proof. Since $c_{0}>0$, and $\nu_{2}$ is bounded on a finite domain,

$$
\nu_{x} \leq \nu(x) \leq \sup _{x \in D \backslash \Gamma_{S}} \nu_{1}(x)+\sup _{x \in D \backslash \Gamma_{S}} \nu_{2}(x)
$$

then

$$
\nu_{x} \leq \frac{1}{2 n h} \sup _{x \in D \backslash \Gamma_{S}}\left\{\nu_{0}(x)+\nu_{h}(x)\right\}+\nu_{2}(\sqrt{3 h})
$$

which is $\mathcal{O}\left(h^{-1}\right)$.

Experimentally, we find that once $X \in \Gamma_{T} \backslash \Gamma_{S}$, the expected number of steps until that path exits is $\mathcal{O}\left(h^{-\alpha(n)}\right)$ where $\alpha(n) \approx 0.44 \cdot n^{-2 / 5}$. This means that as the dimension of the problem increases, the number of steps required to find the boundary once a path has entered the tangent layer $\Gamma_{T}$ becomes less sensitive to the stepsize $h$. We next describe our simulations in the context of the problem (1.3).

\section{Algorithms.}

The probabilistic representation of the solution to the Dirichlet problem

$$
\frac{1}{2} \triangle u(x)+g(x)=0 \quad \text { for } \quad x \in D,\left.\quad u\right|_{\partial D}=\psi(x)
$$

takes the form $u(x)=\mathbb{E}[\psi(X(\tau))+Z(\tau)]$ where $(X(t), Z(t))$ solve the system of stochastic differential equations $(0<t<\tau)$

$$
d X^{i}(t)=d W^{i}(t), \quad i=1, \ldots, n, \quad d Z(t)=g(X(t)) d t
$$

together with the initial conditions $X(0)=x$ and $Z(0)=0$. In $(3.2),\left(W^{1}, \ldots, W^{n}\right)^{T}$ is an $n$-dimensional Brownian motion and $\tau$ is the first exit time of process $X(t)$ from the bounded domain $D[5,10]$.

Repeatedly applying Ito's formula to the system (3.2) and neglecting higher order terms yields the following one step approximation $[6,8]$ :

$$
\begin{aligned}
X^{i}(t+h)= & X^{i}(t)+\underbrace{\int_{t}^{t+h} d W^{i}(s)}_{=I_{(i)}} \quad i=1, \ldots, n \\
Z(t+h)= & Z(t)+g(X(t)) h \\
& +\partial_{i} g(X(t)) \underbrace{\int_{t}^{t+h} \int_{t}^{s_{1}} d W^{i}\left(s_{2}\right) d s_{1}}_{=I_{(i 0)}}+\frac{1}{2} \triangle g(X(t)) \frac{h^{2}}{2}
\end{aligned}
$$

The neglected error in the expansion (3.3) is of weak order 3 [8]. Replacing the integrals $I_{(i)}, I_{(i 0)}$ in (3.3) by the following set of random variables does not change the weak order of accuracy:

$$
I_{(i)} \rightarrow \sqrt{h} \xi^{i}, \quad I_{(i 0)} \rightarrow \frac{h^{\frac{3}{2}}}{2} \xi^{i} \quad i=1, \ldots, n
$$


where $\xi^{i}$ is a three point random variable distributed according to

$$
\xi^{i}=\left\{\begin{array}{rl} 
\pm \sqrt{3} & \text { each with probability } \frac{1}{6} \\
0 & \text { with probability } \frac{2}{3}
\end{array} .\right.
$$

Let $\rho=\rho(x, \partial D)$ be the distance from a point $x \in D$ to the boundary $\partial D$ and introduce the boundary layers $\Gamma_{T}(h)=\{x \in D \mid \rho<\sqrt{3 n h}\}$ and $\Gamma_{S}(h)=\{x \in$ $\left.D \mid \rho<h^{2}\right\}$. Consider the following Markov chain starting in $D$ and stopping in $\partial D$ :

Algorithm 3.1 (Walk on Cubes). Choose $h>0$ and $X_{0}=x \in D \backslash \Gamma_{S}(h)$, set $h_{0}=h$ and get the subsequent elements for $k=0,1, \ldots$ as

if $\left(X_{k} \in \Gamma_{S}(h)\right)$ set $X_{\nu}$ to a point on $\partial D$ closest to $X_{k}$ and $S T O P$

else

$$
\begin{aligned}
h_{k+1} & = \begin{cases}h, & \text { if } X_{k} \in D \backslash \Gamma_{T}(h) \\
\frac{\rho\left(X_{k}, \partial D\right)^{2}}{3 n}, & \text { if } X_{k} \in \Gamma_{T}(h)\end{cases} \\
X_{k+1} & =X_{k}+\sqrt{h_{k+1}} \xi_{k+1}
\end{aligned}
$$

Note that $h_{k+1} \leq h$ for $k=0, \ldots, \nu_{x}-1$. The chain is stopped as soon as $X_{k} \in \Gamma_{S}$, i.e. $k=\nu_{x}$. We obtain a chain $X_{0}, X_{1}, \ldots, X_{\nu_{x}}$ with $X_{k} \in D$ for $0 \leq k<\nu_{x}$. In particular, we can approximate the exit point $X(\tau)$ by $X_{\nu_{x}} \in \partial D$ and the exit time $\tau$ by $h_{1}+h_{2}+\cdots+h_{\nu_{x}}$, where the chain starts at $X_{0}=x$.

Estimating the expectations by finite means over $N$ paths, we propose the following algorithm based on Algorithm 3.1 to find $u_{h}^{N}(x)$ approximating the solution $u(x)$ to problem (3.1) with initial stepsize $h>0$.

Algorithm 3.2 (Monte Carlo for Dirichlet problems). Choose $x \in$ $D \backslash \Gamma_{S}(h)$, set $u=0$ and $h_{0}=h$.

if $\left(x \in \Gamma_{T}\right)$ set $h_{0}=\frac{\rho(X, \partial D)^{2}}{3 n}$

for $p=1, \ldots, N$

- $X=x, Z=0, h=h_{0}$

- for $k=0,1, \ldots$

- generate random n-vector $\xi$ with components distributed according to (3.5)

- update $Z=Z+g(X) h+\partial_{i} g(X) \frac{h^{\frac{3}{2}}}{2} \xi^{i}+\frac{1}{2} \triangle g(X) \frac{h^{2}}{2}$

- if $X \in \Gamma_{S}$ : set $X_{\nu}$ to a point on $\partial D$ closest to $X, S T O P$

else

if $X \in \Gamma_{T}:$ set $h=\frac{\rho(X)^{2}}{3 n}$

else set $h$ to initial stepsize

- update $X=X+h^{\frac{1}{2}} \xi$

- $u=u+\left(\psi\left(X_{\nu}\right)+Z\right)$

Compute expectation $u_{h}^{N}=\frac{1}{N} u$. 


\section{Numerical experiments.}

In this section we present results from extensive numerical experiments on the Algorithm 3.2. We first study in detail the case $g(x) \equiv 1$ and $\psi(x) \equiv 0$ (exit time problem) and present results of an experimental error analysis. We later show results from simulations with a varying inhomogeneity $g_{k}(x)$ consisting of a product of cosines with different frequencies $k^{i}(i=1, \ldots, n)$.

A comparison of Algorithm 3.2 based on Algorithm 3.1 is made with other algorithms that have appeared in the literature.

The $n$-sphere with radius 1 and center in the origin is denoted $S_{1}^{n}=\{x \in$ $\left.\mathbb{R}^{n}|| x \mid<1\right\}$, and its boundary is $\partial S_{1}^{n}$.

\subsection{Experimental setup.}

Algorithm 3.2 has two parameters influencing its accuracy: the initial stepsize $h$ and the number of simulated paths $N$. The approximate solution $u_{h}^{N}$ and the error $\left|u-u_{h}^{N}\right|$ are random variables themselves, varying both for different values of $h$ and $N$. In order to get consistent experimental results, we chose the following strategy to improve the estimate for a certain outcome $o$. We computed the mean $\mathbb{E}[o]$ for $N_{S}$ simulations having outcomes $o^{(1)}, \ldots, o^{\left(N_{S}\right)}$ by $\bar{o}_{N_{S}}=N_{S}^{-1} \sum_{i=1}^{N_{S}} o^{(i)}$. To check the consistency we used this procedure: an $f \in(0,1)$ was chosen to compare to a $95 \%$ confidence interval computed from the standard deviation $S_{N_{S}}[o]=\sqrt{\operatorname{var}[o]}($ see 4.1$)$.

\section{Algorithm 4.1 .}

- Make at least $N_{S}>1$ independent simulations.

- while $\left(2 S_{N_{S}}[o] \geq f \bar{o}_{N_{S}}\right)$ make another simulation, $N_{S}=N_{S}+1$.

- take $\bar{o}_{N_{S}}$ as an estimate for $\mathbb{E}[o]$.

In Algorithm 4.1, the mean is considered reliable if its (numerical) 95\%confidence interval is smaller than its value by the factor $f$. A smaller $f$ implies a more reliable estimate for $\mathbb{E}[o]$. The standard deviation of $o, S_{N_{S}}[o]$, is the numerical estimate

$$
S_{N_{S}}^{2}[o]=\left(\frac{D_{N_{S}}[o]}{N_{S}-1}\right)^{\frac{1}{2}}, \quad D_{N_{S}}[o]=N_{S}^{-1} \sum_{i=1}^{N_{S}}\left(o^{(i)}\right)^{2}-\left(N_{S}^{-1} \sum_{i=1}^{N_{S}} o^{(i)}\right)^{2} .
$$

\subsection{Experimental error analysis for the exit time problem.}

We first studied a simplest case of problem (3.1), namely $g \equiv 1$ and $\psi \equiv 0$. The solution is $u(x)=\frac{1}{n}\left(1-|x|^{2}\right)$. Furthermore, $u(0)=\mathbb{E} \tau$ where $\tau$ is the first exit time of an $n$-dimensional Brownian motion $X$ from $S_{1}^{n}$.

We calculated an approximation for $u(0)$ and looked at the absolute error $e_{a}=\left|u(0)-u_{h}^{N}(0)\right|$. For the relative error $e_{r}=e_{a} / u(0)$ we have $e_{r}=n e_{a}$. When applying Algorithm 3.2 to this problem, two errors arise: the systematic one $e_{S y}$ by replacing the first $n$ equations of system (3.2) by the discrete Algorithm 3.1, 
and a statistical error, $e_{S t}$, due to a finite sample of $N$ paths which replaces the expectation $\mathbb{E}$ by a mean of the $N$ sample. Both contribute to the absolute error $e_{a}=e_{S y}+e_{S t}$. This section hopefully illuminates the behavior of these errors. In all cases, we made at least 30 simulations and used a confidence factor of $f=0.1$ or $f=0.05$ (see Algorithm 4.1).

Figure 4.1 shows the absolute error $e_{a}$ from a simulation with $N=10^{4}$ paths. A simulation with $N=5 \times 10^{3}$ paths looks very similar but with slightly bigger statistical errors.

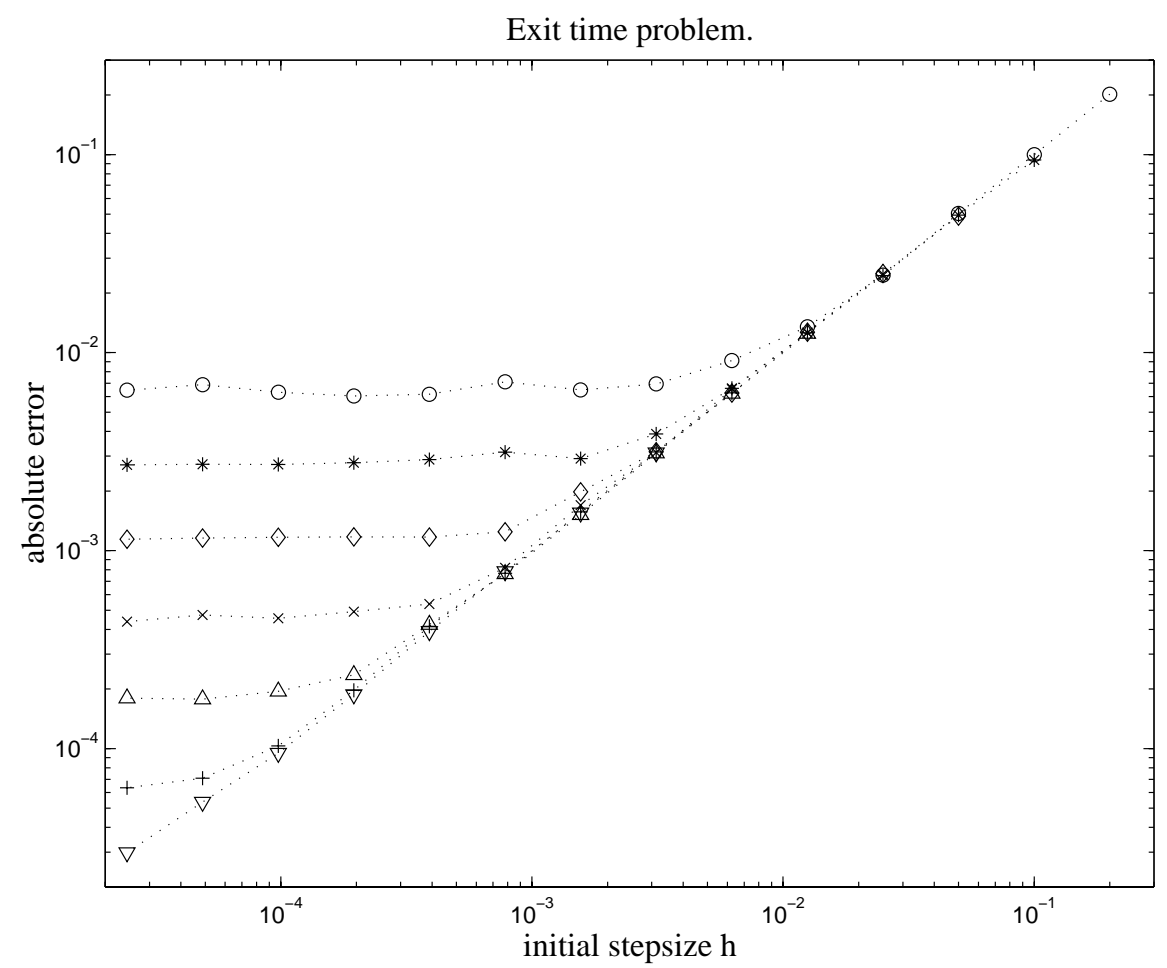

Figure 4.1: Absolute error $\left|u(0)-u_{h}^{N}(0)\right|$ versus initial stepsize $h$ when applying Algorithm 3.2 to the problem (3.1) with $g=1, \psi=0$ in $D=S_{1}^{n}$. Parameters are $f=0.1$ and $N=10^{4}$. Following dimensions are shown: $n=1(\circ), 2(*), 4(\diamond), 8(\times), 16(\triangle), 32(+), 64(\nabla)$.

We now examine the behavior of the numerical standard deviation $S_{N}\left[u_{h}^{N}\right]$ of solution $u_{h}^{N}$ (see 4.1 with $o_{i}=\tau_{h}^{(i)}$ and $N_{S}=N$, where $\tau_{h}^{(i)}$ is the estimate of the exit time of path $i$ ).

Table 4.1 shows that the standard deviation $S_{N}$ depends very weakly on the initial stepsize $h$ for a fixed dimension $n$ and number of paths $N$.

Comparing the absolute error $e_{a}$ with $S_{N}$ for sufficiently small initial stepsize $h$ in Table 4.2, we see that the statistical error is proportional to $S_{N}$ and is thus 
Table 4.1: Numerical standard deviation $S_{N}\left[u_{h}^{N}\right]$ versus stepsize $h$ for different dimensions $n$ and $N=10^{4}$ paths.

\begin{tabular}{c|cccc}
\hline \multirow{2}{*}{$n$} & \multicolumn{4}{|c}{$S_{N}\left[u_{h}^{N}\right]$} \\
\cline { 2 - 5 } & $h=0.025$ & $h=0.0125$ & $h=0.00625$ & $h=0.003125$ \\
\hline 1 & $8.153 \mathrm{e}-3$ & $8.184 \mathrm{e}-3$ & $8.183 \mathrm{e}-3$ & $8.178 \mathrm{e}-3$ \\
2 & $3.538 \mathrm{e}-3$ & $3.539 \mathrm{e}-3$ & $3.533 \mathrm{e}-3$ & $3.531 \mathrm{e}-3$ \\
4 & $1.444 \mathrm{e}-3$ & $1.446 \mathrm{e}-3$ & $1.448 \mathrm{e}-3$ & $1.446 \mathrm{e}-3$ \\
8 & $5.593 \mathrm{e}-4$ & $5.597 \mathrm{e}-4$ & $5.623 \mathrm{e}-4$ & $5.598 \mathrm{e}-4$ \\
\hline
\end{tabular}

proportional to $1 / \sqrt{N}$ as expected. This is important: the statistical error has the same dependence on $N$, regardless of the dimension $n$.

Table 4.2: Comparison of $S_{N}\left[u_{h}^{N}\right]$ and absolute error $e_{a}$ for different dimensions $n$ and different stepsizes $h$ (again $N=10^{4}$ paths).

\begin{tabular}{c|ccc|ccc}
\hline & \multicolumn{3}{|c|}{$h=0.1 \cdot 2^{-11}$} & \multicolumn{3}{c}{$h=0.1 \cdot 2^{-12}$} \\
\cline { 2 - 7 } $\mathrm{n}$ & $e_{a}$ & $S_{N}$ & $e_{a} / S_{N}$ & $e_{a}$ & $S_{N}$ & $e_{a} / S_{N}$ \\
\hline 1 & $6.885 \mathrm{e}-3$ & $8.152 \mathrm{e}-3$ & 0.845 & $6.457 \mathrm{e}-3$ & $8.172 \mathrm{e}-3$ & 0.790 \\
2 & $2.726 \mathrm{e}-3$ & $3.531 \mathrm{e}-3$ & 0.772 & $2.711 \mathrm{e}-3$ & $3.540 \mathrm{e}-3$ & 0.766 \\
4 & $1.158 \mathrm{e}-3$ & $1.443 \mathrm{e}-3$ & 0.803 & $1.141 \mathrm{e}-3$ & $1.443 \mathrm{e}-3$ & 0.791 \\
8 & $4.731 \mathrm{e}-4$ & $5.584 \mathrm{e}-4$ & 0.847 & $4.378 \mathrm{e}-4$ & $5.587 \mathrm{e}-4$ & 0.784 \\
\hline
\end{tabular}

Table 4.3 shows the numerical standard deviation $S_{N}\left[u_{h}^{N}\right]$ for various dimensions $n$ and two sample sizes, $N=10^{4}$ and $N=5 \times 10^{3}$, at fixed initial stepsize $h$. Additionally, we added the numerical slope of a graph of $S_{N}\left[u_{h}^{N}\right](n)$ in a double logarithmic plot, given by

$$
\text { slope }=\frac{\log \frac{S_{i}}{S_{i+1}}}{\log \frac{n_{i}}{n_{i+1}}}
$$

To summarize, we find the following behavior for the absolute error when applying Algorithm 3.2 to the exit time problem:

$$
e_{a}=C_{s y} h+C_{s t} \frac{n^{-s}}{\sqrt{N}}
$$

In Equation (4.2), $C_{s y}$ is independent of $h$ (Figure 4.1) and $N$ (similar Figure to Figure 4.1 for $N=5 \times 10^{3}$ paths), $C_{s t}$ is independent of $h$ (Table 4.1) and $N$ (Table 4.2) and further $s=s(n)>1$ (Table 4.3). This is an encouraging 
Table 4.3: $S_{N}\left[u_{h}^{N}\right]$ versus dimension $n$ for two sample sizes at fixed initial stepsizes $h=1 / 320$ and $h=1 / 5120$.

\begin{tabular}{r|cc|cc||cc|cc}
\hline & \multicolumn{4}{|c||}{$h=1 / 320$} & \multicolumn{3}{c}{$h=1 / 5120$} \\
& \multicolumn{2}{|c|}{$N=10000$} & $N=5000$ & \multicolumn{2}{c}{$N=10000$} & \multicolumn{2}{c}{$N=5000$} \\
$\mathrm{n}$ & $S_{N}$ & slope & $S_{N}$ & slope & $S_{N}$ & slope & $S_{N}$ & slope \\
\hline 1 & $3.271 \mathrm{e}-2$ & -1.21 & $4.620 \mathrm{e}-2$ & -1.21 & $3.264 \mathrm{e}-2$ & -1.21 & $4.619 \mathrm{e}-2$ & -1.21 \\
2 & $1.412 \mathrm{e}-2$ & -1.29 & $1.999 \mathrm{e}-2$ & -1.29 & $1.413 \mathrm{e}-2$ & -1.29 & $2.001 \mathrm{e}-2$ & -1.29 \\
4 & $5.782 \mathrm{e}-3$ & -1.37 & $8.168 \mathrm{e}-3$ & -1.36 & $5.772 \mathrm{e}-3$ & -1.37 & $8.164 \mathrm{e}-3$ & -1.37 \\
8 & $2.239 \mathrm{e}-3$ & -1.43 & $3.178 \mathrm{e}-3$ & -1.44 & $2.240 \mathrm{e}-3$ & -1.43 & $3.164 \mathrm{e}-3$ & -1.43 \\
16 & $8.316 \mathrm{e}-4$ & -1.45 & $1.176 \mathrm{e}-3$ & -1.46 & $8.322 \mathrm{e}-4$ & -1.45 & $1.178 \mathrm{e}-3$ & -1.46 \\
32 & $3.038 \mathrm{e}-4$ & -1.49 & $4.290 \mathrm{e}-4$ & -1.48 & $3.037 \mathrm{e}-4$ & -1.48 & $4.286 \mathrm{e}-4$ & -1.48 \\
64 & $1.085 \mathrm{e}-4$ & & $1.538 \mathrm{e}-4$ & & $1.087 \mathrm{e}-4$ & & $1.536 \mathrm{e}-4$ & \\
\hline
\end{tabular}

result because it implies that the statistical error retains the $\mathcal{O}\left(N^{-1 / 2}\right)$ behavior expected of Monte-Carlo simulation, but the coefficient of this term actually decreases with $n$.

\subsection{Comparison of different algorithms.}

We now show our results which compare Algorithm 3.2 with other probabilistic methods of solving problem (3.1) appearing in the literature. We chose an $n=3$ dimensional problem and the domain $D=S_{1}^{3}$.

\subsubsection{Comparing algorithms.}

For convenience, we summarize the basic features of all algorithms discussed in (4.3-4.5). To simplify notation we define the increments as

$$
\Delta X=X_{k+1}-X_{k} \quad \text { and } \quad \Delta Z=Z_{k+1}-Z_{k}
$$

and neglect subscripts $k+1$ also on $h, r$ and $\xi$. Using this notation, the algorithms take the following form:

- simplest random walk [9]:

$$
\begin{aligned}
\Delta X & =\sqrt{h} \xi \\
\Delta Z & =g(X) h
\end{aligned}
$$

- walk on cubes (see Algorithm 3.1):

$$
\begin{aligned}
\Delta X & =\sqrt{h} \xi \\
\Delta Z & =g(X) h+\partial_{i} g(X) \frac{h^{\frac{3}{2}}}{2} \xi^{i}+\frac{1}{2} \Delta g(X) \frac{h^{2}}{2}
\end{aligned}
$$


- walk on spheres of global order $h \propto r^{2}$ [10]:

$$
\begin{aligned}
\Delta X & =r \xi \\
\Delta Z & =g(X) \frac{r^{2}}{n}
\end{aligned}
$$

- walk on spheres of global order $h^{2} \propto r^{4}$ [10]:

$$
\begin{aligned}
\Delta X & =r \xi \\
\Delta Z & =g(X) \frac{r^{2}}{n}+\partial_{i} g(X) \frac{r^{2}}{2(n+2)} r \xi^{i}+\frac{1}{2} \triangle g(X) \frac{4+n}{n^{2}(2+n)} r^{4}
\end{aligned}
$$

In Table 4.4 the random variables, width of boundary layers $\Gamma_{T}\left(\Gamma_{S}\right)$ denoted by $\rho_{T}\left(\delta_{S}\right)$ and the global order of convergence as predicted by theory are summarized for all four algorithms.

Table 4.4: Random variables, boundary layers and global order of convergence for the algorithms compared.

\begin{tabular}{c|c|cc|c}
\hline Algorithm & random variables & $\rho_{T}$ & $\delta_{S}$ & order \\
\hline$(4.3)$ & $\xi^{i}= \pm 1$ with probability $\frac{1}{2}$ & $\sqrt{n h}$ & $h$ & $h$ \\
3.1 & $\xi^{i}$ as in $(3.5)$ & $\sqrt{3 n h}$ & $h^{2}$ & $h^{2}$ \\
$(4.4)$ & $\xi$ uniform on $\partial S_{1}^{n}$ & $r$ & $r^{2}$ & $r^{2}$ \\
$(4.5)$ & & $r$ & $r^{4}$ & $r^{4}$ \\
\hline
\end{tabular}

For the algorithms 3.1 and (4.3) we used the ran3 random number generator [12] to generate the $\xi^{i}$, while the random variables uniform on $\partial S_{1}^{n}$ for algorithms $(4.4,4.5)$ were generated using normal random variables from the Blitz++ library $[13,14]$ and scaling the resulting vectors to unit length. We always chose $D=S_{1}^{n}$ for the domain.

In order to compare the convergences, we set $h=\frac{r^{2}}{n}$.

\subsubsection{Exit time problem.}

In Section 4.2 we found a truncation error of order $h$ for Algorithm 3.2, whereas theoretically order $h^{2}$ should be achieved.

Figure 4.2 shows that the algorithm (4.5) also does not achieve the predicted rate of convergence (order $h^{2}=r^{4}$ ). Instead its convergence rate is of the same order as that of walk-on-cubes, Algorithm 3.2. The simplest random walk (4.3) has the same order of convergence (it achieves the predicted order $h$ ) whereas the simpler walk on spheres (4.4) converges immediately. It should be noted, that for this simple problem, fewer steps are better (the stopping layer $\Gamma_{S}$ is wider for the low order algorithms, see Table 4.4). 


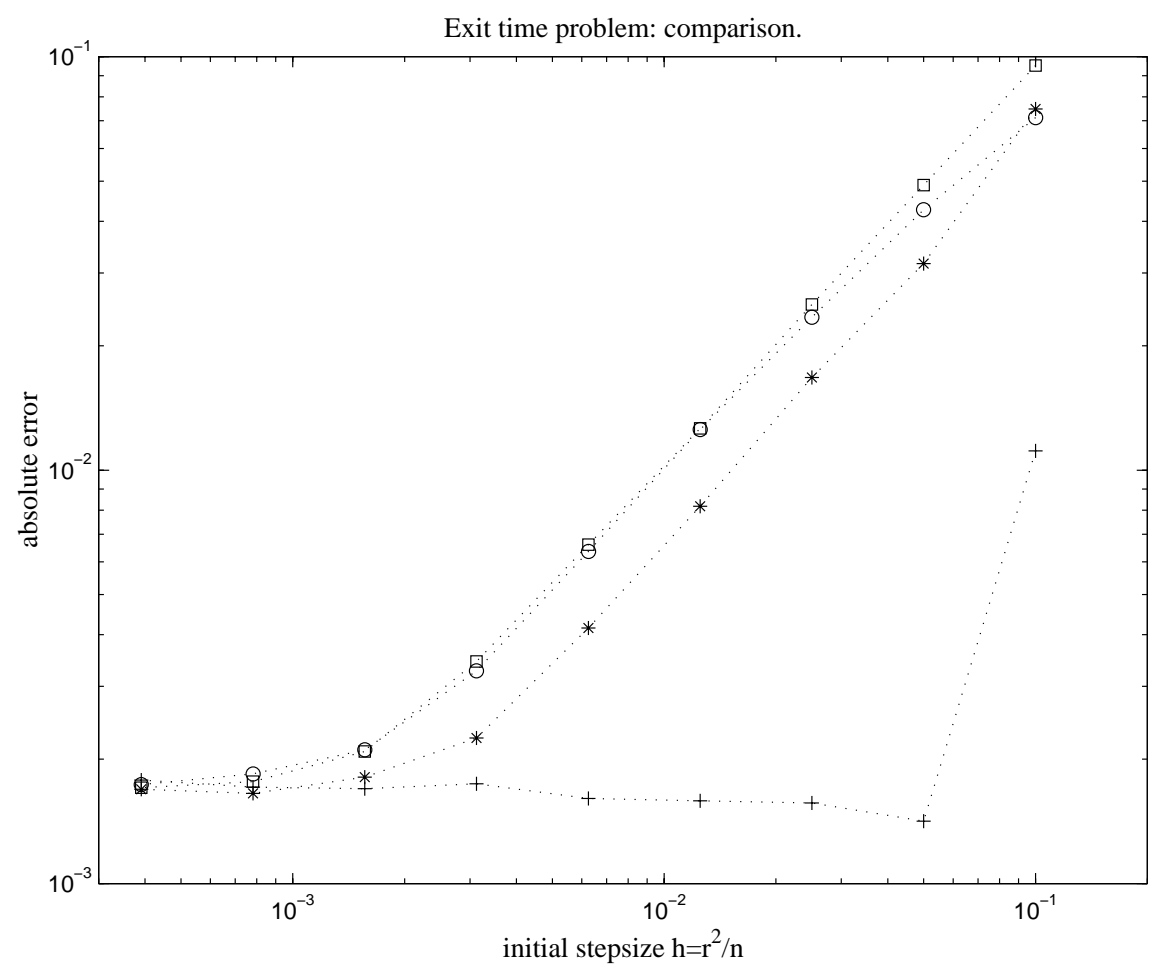

Figure 4.2: Absolute error $\left|u(0)-u_{h}^{N}(0)\right|$ versus initial stepsize $h=\frac{r^{2}}{n}$ when applying Algorithms 3.2 and $(4.3,4.4,4.5)$ to the problem (3.1) with $g=1, \psi=0$. Parameters are $f=0.05, N=10^{4}$ and the dimension is $n=3$. $\square$ is Algorithm $3.1, \circ$ is $(4.5), *$ is $(4.3)$ and + is $(4.4)$.

\subsubsection{Varying source term $g(x)$}

In this section we consider problem (3.1) with

$$
g(x)=\prod_{i=1}^{n} \cos \left(2 \pi k^{i} x^{i}\right), \quad k^{i}=1,2, \ldots, \quad k=\left(k^{1}, \ldots, k^{n}\right) .
$$

A solution is easily found to be $u(x)=g(x) /\left(2 \pi^{2}|k|^{2}\right)$ which we assigned as boundary condition $\psi(x)$ to get uniqueness on the surface of the hypersphere.

From Figure 4.3 we see that for a harder problem (right plot) with higher frequencies $k^{i}$, higher order algorithms show better performance than low order algorithms. Furthermore, Algorithm 3.2 shows better convergence than algorithm (4.5). 

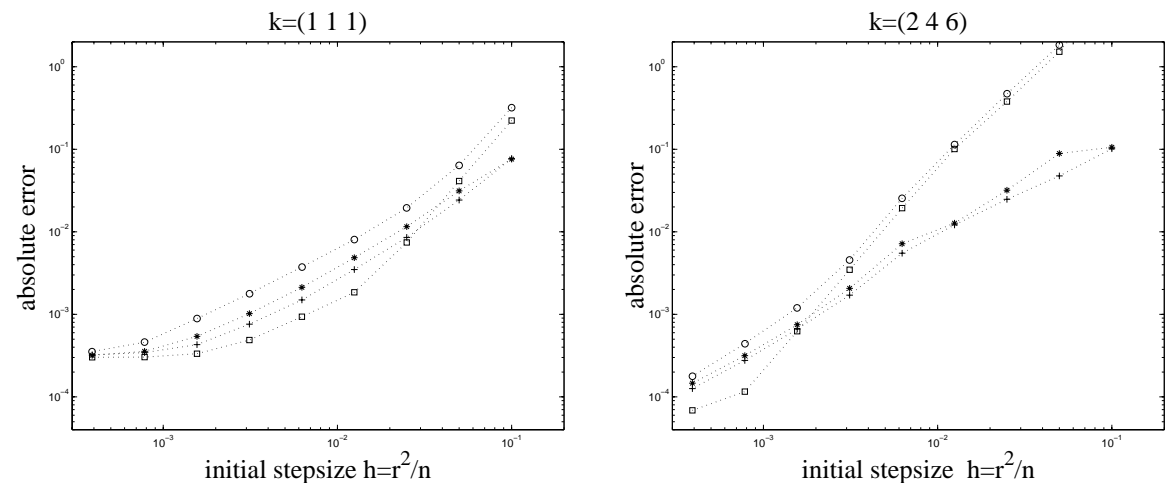

Figure 4.3: Absolute error $\left|u(0)-u_{h}^{N}(0)\right|$ versus initial stepsize $h=\frac{r^{2}}{n}$ when applying Algorithms 3.2 and $(4.3,4.4,4.5)$ to the problem (3.1) with $g(x)$ from (4.6) with $k=(1,1,1)$ (left) and $k=(2,4,6)$ (right). Parameters are $f=0.05$, $N=10^{4}$ and the dimension is $n=3$. $\square$ is Algorithm 3.1, ○ is (4.5), * is (4.3) and + is (4.4).

\section{Generalizations.}

The generalization of Algorithm 3.2 to the more general Dirichlet problem (1.1)

$$
\frac{1}{2} \triangle u(x)+b^{i}(x) \partial_{i} u(x)+c(x) u(x)+g(x)=0 \quad x \in D,\left.u\right|_{\partial D}=\psi(x)
$$

with $c(x) \leq 0$ is straight forward: Instead of system (3.2) consider the system [10],

$$
\begin{aligned}
d X^{i}(t) & =d W^{i}(t), \quad i=1, \ldots, n \\
d Y(t) & =c(X) Y d t+b^{i}(X) Y d W^{i}(t) \\
d Z(t) & =Y g(X) d t
\end{aligned}
$$

together with the initial conditions $X(0)=x, Y(0)=1$ and $Z(0)=0$. Then the probabilistic representation of the solution to problem (5.1) is $u(x)=\mathbb{E}[\psi(X(\tau)) Y(\tau)+$ $Z(\tau)]$. Analogous to (3.3), we introduce

$$
\begin{aligned}
\gamma^{i j}(x) & =\partial_{i} b^{j}(x)+b^{i}(x) b^{j}(x) \\
\gamma^{i 0}(x) & =\partial_{i} c(x)+b^{i}(x) c(x) \\
\gamma^{0 i}(x) & =y \frac{1}{2} \triangle b^{i}(x)+\left\langle b(x), \nabla b^{i}(x)\right\rangle+c(x) b^{i}(x) \\
\gamma^{00}(x) & =\frac{1}{2} \triangle c(x)+c^{2}(x)+\langle b(x), \nabla c\rangle \\
\zeta^{i 0}(x) & =\partial_{i} g(x)+b^{i}(x) g(x) \\
\zeta^{00}(x) & =\frac{1}{2} \triangle g(x)+\langle b(x), \nabla g\rangle+c(x) g(x)
\end{aligned}
$$


where $\langle\cdot, \cdot\rangle$ denotes the standard scalar product in $\mathbb{R}^{n}$. Making further use of the identity

$$
\int_{t}^{t+h} \int_{t}^{s_{1}} d s_{2} d W^{i}\left(s_{1}\right)=h \int_{t}^{t+h} d W^{i}(s)-\int_{t}^{t+h} \int_{t}^{s_{1}} d W^{i}\left(s_{2}\right) d s_{1}
$$

we get the following one step approximation for Algorithm 3.2:

$$
\begin{aligned}
& Z=Z+ Y\left(g(X) h+\zeta^{i 0}(X) \frac{h^{\frac{3}{2}}}{2} \xi^{i}+\zeta^{00}(X) \frac{h^{2}}{2}\right) \\
&(5.3) Y=Y\left(1+\left(b^{i}(X)+\gamma^{0 i}(X) h\right) h^{\frac{1}{2}} \xi^{i}+c(X) h+\gamma^{i j}(X) \frac{h}{2}\left(\xi^{i} \xi^{j}+\Xi^{i j}\right)\right. \\
&\left.+\left(\gamma^{i 0}(X)-\gamma^{0 i}(X)\right) \frac{h^{\frac{3}{2}}}{2} \xi^{i}+\gamma^{00}(X) \frac{h^{2}}{2}\right)
\end{aligned}
$$

In (5.3), $\xi^{i}$ is as in (3.5) and and the $(n \times n)$-array $\Xi^{i j}$ is distributed according to the law $[6]$

$$
\mathbb{P}\left[\Xi^{i i}=-1\right]=1, \quad \mathbb{P}\left[\Xi^{i j}= \pm 1\right]=\frac{1}{2} \quad \text { for } \quad i<j .
$$

Note that $\Xi$ is skew symmetric w.r.t. the off-diagonal elements $\left(\Xi^{i j}=-\Xi^{j i}, i, j=\right.$ $1, \ldots, n, j \neq i$ ) with -1 on the diagonal. For this skew symmetric part, any higher order bounded approximation to gaussian random numbers is adequate.

\section{Conclusions.}

From the examples of two Poisson problems with Dirichlet boundary conditions in hyperspherical domains, we have seen that Monte-Carlo simulations of stochastic differential equations can indeed give very satisfactory results for the Dirichlet problem in $n$ dimensions. From our examples, we also saw that it is not difficult for the random walk, which uses bounded approximations for the Brownian increments, to find the boundary. Furthermore, as the dimension of the space is increased, not only does the canonical $\mathcal{O}\left(N^{-1 / 2}\right)$ statistical error remain valid, but the coefficient of $N^{-1 / 2}$ actually seems to decrease as the dimension $n$ increases. We think this is an encouraging result. As $n$ increases, termination of the walk at the boundary also seems to become less sensitive to the initial step size. This conclusion seems valid for each of the methods tested: a simple walk with $\Delta X_{i}= \pm \sqrt{h}$ for each coordinate $i$, but also the higher order methods walk on spheres (see [7]) and walk on cubes (see Algorithm 3.1). The global order of accuracy is sometimes not as theoretically predicted (see Table 4.4).

However, there is reason to believe the three models (above) for the increments of Brownian motion have weaknesses. In one dimension, the walk on spheres procedure is the same as the simple 2-point method (simple random walk). The walk on cubes increment, which has an advantage that it lends itself readily to Runge-Kutta approaches $([6],[8],[11])$, is not isotropically distributed in 
$n$-space. Thus none of these models has all the properties we would like. The walk on spheres increment is isotropically distributed, but the radial distribution doesn't model the $p \sim \exp \left(-r^{2} /(2 h)\right) r^{n-1}$ radial density well. Walk on cubes has a somewhat better radial distribution, but isn't isotropic. Although in a few cases the global order is the expected $\mathcal{O}\left(h^{2}\right)$, in others it is only $\mathcal{O}(h)$. This is clearly better than the crude estimate $\mathcal{O}\left(h^{1 / 2}\right)$ with an unsophisticated boundary finding algorithm, however. It is our hope that better bounded models for Brownian increments will show improved accuracy. The current results, however, have to be considered stimulating: higher dimensional problems do not seem any less tractable than lower dimensional ones as we see at the end of Subsection 2.3 and in Table 4.3 .

\section{Acknowledgement.}

Both authors would like to thank Grigori Milstein for very helpful discussions.

\section{REFERENCES}

1. M. Abramowitz and I. Stegun, Handbook of Mathematical Functions, Dover Publications, 1972, or Nat. Bureau of Standards edition, 1964.

2. J. L. Doob, Classical Potential Theory and its Probabilistic Counterpart, Springer Verlag, 1984.

3. E. B. Dynkin, Markov Processes (eng. translation) Springer Verlag, 1965.

4. A. Erdélyi, Bateman Manuscript Project: Higher Transcendental Functions, vol II, Robert E. Krieger Publ. Co., 1981. see Chapter XI.

5. M. Freidlin, Functional Integration and Partial Differential Equations, Study 109, Annals of Mathematics Studies, Princeton Univ. Press, 1985.

6. P. E. Kloeden, E. Platen, Numerical Solution of Stochastic Differential Equations, Application of Mathematics, 23, Springer-Verlag, Berlin, 1992

7. G. N. Milstein and N. F. Rybkina, An Algorithm for random walks over small ellipsoids for solving the general Dirichlet problem, Computational Mathematics and Mathematical Physics, Vol. 33, No. 5, pp. 631-647, 1993.

8. G. N. Milstein, Numerical Integration of Stochastic Differential Equations, Mathematics and Its Applications, Kluwer Academic Publishers, Dordrecht, 1995

9. G. N. Milstein, The simplest random walk for general dirichlet problems, Talk at Stochastic Numerics Conference 2001 at ETH

10. G. N. Milstein, Weak approximation of a diffusion process in a bounded domain Stochastics and Stochastic Reports, Vol. 62, pp. 147-200, 1997.

11. W. P. Petersen, A General implicit splitting for numerical simulation of stochastic differential equations, SIAM Journal on Num. Analysis, Vol. 35, No. 4, pp. 14391451, Aug. 1998.

12. W. H. Press, Numerical Recipes in C: the Art of Scientific Computing, Cambridge University Press, Cambridge, 1999 
13. T. L. Veldhuizen, Arrays in Blitz++, in Proceedings of the 2nd International Scientific Computing in Object-Oriented Parallel Environments (ISCOPE'98), SpringerVerlag, 1998

14. T. L. Veldhuizen, The Blitz++ user guide. http://oonumerics.org/blitz/

15. G. N. Watson, A Treatise on the Theory of Bessel Functions, 2nd ed., Cambridge Univ. Press, 1958.

16. A. D. Wentzell, Theorie zufälliger Prozesse, Birkhäuser Verlag, 1978. 